\title{
On Public Values and Power Indices
}

\author{
Cesarino Bertini*, Izabella Stach**
}

\begin{abstract}
In this paper, we analyze some values and power indices from a different point of view that are well-defined in the social context where the goods are public. In particular, we consider the Public Good index (Holler, 1982), the Public Good value (Holler and Li, 1995), the Public Help index (Bertini et al., 2008), the König and Bräuninger index (1998) also called the Zipke index (Nevison et al., 1978), and the Rae index (1969). The aims of this paper are: to propose an extension of the Public Help index to cooperative games; to introduce a new power index with its extension to a game value; and to provide some characterizations of the new index and values.
\end{abstract}

Keywords: cooperative game theory, simple game, values, public values, power indices, public power indices

Mathematics Subject Classification: 91A06, 91A12, 91B12

Revised: April 14, 2015

\section{INTRODUCTION}

A value for $n$-person cooperative games is a function able to represent a reasonable expectation of the sharing of global winnings amongst the players. A power index is a value for a particular class of games called simple games. The power indices approach is widely used to measure a priori voting power of members of a committee. The concept of value was introduced for the first time by Lloyd Stowell Shapley in (1953). The following year, Shapley and Martin Shubik introduced the "Shapley and Shubik power index" (Shapley and Shubik, 1954). Since 1954, numerous remarkable power indices have been introduced in the literature for simple games. These power indices are based on diverse bargaining models and/or axiomatic assumptions. Some indices have been derived from existing values; i.e., the Shapley and Shubik (1954) as well as the Banzhaf (1965) and the Coleman (1971). Other power indices were formulated exclusively for simple games; i.e., the Public Good index (Holler, 1982), the Deegan

* University of Bergamo, Department of Management, Economics and Quantitative Methods, Italy, e-mail: cesarino.bertini@unibg.it

** AGH University of Science and Technology, Faculty of Management, Poland, e-mail: istach@zarz.agh.edu.pl, corresponding author 
and Packel index (1978), and the Johnston index (1978). In this paper, we analyze some values and power indices well-defined in the social context where the goods are public; e.g., the Public Good index, the Public Good value, the Public Help index (Bertini et al., 2008), the König and Bräuninger index (1998) also called the Zipke index (Nevison et al., 1978), and the Rae index (1969). We also introduce an extension of the Public Help index as a game value and a new power index with its extension as a game value. Some properties of the new proposed index and values are given.

The paper is organized as follows. Section 2 presents notations and preliminary definitions that refer to cooperative games, simple games, and several properties of power indices. The power indices considered in this paper, as well as a new proposed power index, are described in Section 3. Section 4 is devoted to comparing the considered power indices from the point of view of some desirable properties. Section 5 presents the normalized and absolute Public good value and the propositions of the extension of the Public Help index, as well as a new index to the game value. The paper ends with Section 6 devoted to concluding remarks and further developments. The appendix, at the end of the paper, contains proof of the identity that serves to demonstrate that the new index proposed in this paper satisfies the dominance property.

\section{NOTATIONS AND PRELIMINARY DEFINITIONS}

Let $N=\{1,2, \ldots, n\}$ be a finite set of players. Any subset $S \subseteq N$ is called a coalition, $N$ is called the grand coalition, and $\emptyset$ is called an empty coalition. By $|S|$, we denote the number of members of $S$ : therefore; e.g., $|N|=n$. A cooperative game is a pair $(N, v)$ where $v: 2^{N}>R$, the characteristic function, is a real-valued function from the set of all possible coalitions of players of $N$ to the real number set such that $v(\emptyset)=0$. For every coalition $S, v(S)$ is called the worth of $S$. A cooperative game $v$ is monotonic if $v(S) \leq v(T)$ if $S \subset T \subseteq N$.

If $v$ takes values only in the set $\{0,1\}$, then it is called a simple monotonic game. By $S_{N}$, we denote the set of all simple monotonic games on $N$.

A player $i \in S$, in a simple game $v$, is crucial or pivotal, for the coalition $S$, if $v(S)=1$ and $v(S \backslash\{i\})=0$.

In a simple game, coalitions $S$ with $v(S)=1$ are called winning coalitions and coalitions with $v(S)=0$ losing coalitions. By $W$ (or $W(v)$ ), we denote the set of all winning coalitions, and by $W_{i}$, we denote the set of all winning coalitions to which player $i$ belongs.

If a player does not belong to any winning coalition, then he is called a zero player. A null game is a simple game such that $v(S)=0 \forall S \subseteq N$. Naturally, in any null game, each player is a zero player.

In a minimal winning coalition, all players are crucial. By $W^{m}$ or $W^{m}(v)$, we denote the set of all minimal winning coalitions in $v$, and by $W_{i}^{m}$, we denote the set of all minimal winning coalitions to which player $i$ belongs.

Either the family of winning coalitions $W$ or the subfamily of minimal winning coalitions $W^{m}$ determines the game. 
If a player is not contained in any minimal winning coalition (i.e. $i \notin S \forall S \in W^{m}$ ), then he is called a null player.

A weighted game (also called a weighted majority game), $\left[q ; w_{1}, \ldots, w_{n}\right]$ is a simple game $v \in S_{N}$ with real weights $w_{i} \geq 0 \forall i \in N$ and a non-negative quota $q, \frac{\sum_{i \in N} w_{i}}{2}<$ $q \leq \sum_{i \in N} w_{i}$, such that $v(S)=1 \Leftrightarrow w(S)=\sum_{i \in S} w_{i} \geq q$.

A value is a function $f$ that assigns a payoff distribution $f(v) \in R^{n}$ to every cooperative game $v$. The real number $f_{i}(v)$ is the "value" of the player $i \in N$ in the game $v$ according to $f$.

A power index is a function $f: S_{N}>R^{n}$ that assigns to any simple game $v$ vector $f(v)=\left(f_{1}(v), f_{2}(v), \ldots, f_{n}(v)\right)$ (or equivalently $f(W)=\left(f_{1}(W), f_{2}(W), \ldots, f_{n}(W)\right)$ ). The non-negative real number $f_{i}(v)$ (or $\left.f_{i}(W)\right)$ is interpreted as a "power" of the corresponding player $i \in N$.

There are some properties that are desirable postulates of power indices. Below, we quote only: efficiency, non-negativity, null player, symmetry, dominance, and bloc properties.

If $\sum_{i \notin N} f_{i}(v)=1$ for all $v \in S_{N}$, we said that power index $f$ satisfies the efficiency postulate. A power index $f$ satisfies the non-negativity postulate (or positivity postulate) if $f_{i}(v) \geq 0$ for each $i \in N$ and any $v \in S_{N}$. A power index $f$ satisfies the null player postulate if $f_{i}(v)=0$ for each null player $i \in N$ and all $v \in S_{N}$. If for all $v \in S_{N}$ and for each $i \in N$ and each permutation $\pi: N \rightarrow N f_{i}(v)=f_{\pi(i)}(\pi(v))$ where $(\pi(v))(S)=v\left(\pi^{-1}(S)\right)$, then we said that power index $f$ satisfies the symmetry postulate (also called anonymity postulate). Let $v:\left[q ; w_{1}, \ldots, w_{n}\right]$ be an arbitrary weighted game. A power index $f$ satisfies the dominance (or local monotonicity) postulate if $w_{i} \geq w_{j} \Rightarrow f_{i}(v) \geq f_{j}(v)$ for any distinct players $i, j \in N$. Note that, in the literature for simple games, there is also a stronger version of dominance property (called D-dominance or strong dominance) than is presented here; see, for example, (Felsenthal and Machover, 1995; Bertini et al., 2013a).

Consider a weighted game $W:\left[q ; w_{1}, \ldots, w_{n}\right]$. Let $i$ and $j$ be two distinct players in $W$ and $j$ is not null. If players $i$ and $j$ form a bloc $i \& j$ (i.e., a new entity not belonging to $N$ ) and operate as a single player, then a new game arises which we denote by $W[i \& j]$. The new game $W[i \& j]$ is obtain from $W$ by removing two players $i$ and $j$ and introducing a new player representing the bloc $i \& j$. The quota $q$ stays as there was in $W$. Any player $k \in N \backslash\{i, j\}$ is also a player in $W[i \& j]$ with the same weight, and the weight of the bloc is equal to the sum of the weights of players $i$ and $j$; i.e., $w_{i \& j}=\left(w_{i}+w_{j}\right)$. A power index $f$ satisfies the bloc property if $f_{i \& j}(W[i \& j]) \geq f_{i}(W)$.

\section{POWER INDICES}

In this section, we recall the definitions of the Public Good index, the Public Help index, the König and Bräuninger index, and the Rae index. In Section 3.5, we introduce a new index. The indices considered here are based on winning or minimal winning coalitions and were originally formulated only for simple games.

Henceforth, all the games considered are monotonic and not null. 


\subsection{THE RAE INDEX}

The Rae index, $R$, was introduced by Rae in (1969). The Rae index of a simple game $W$ for player $i$ is defined as follows:

$$
R_{i}(W)=\frac{|\{S: i \in S \in W\}|}{2^{n}}+\frac{|\{S: i \notin S \notin W\}|}{2^{n}}
$$

We remark that this index is equivalent to the Brams and Lake index (1978); see also (Nevison, 1979; Mercik, 1997). There is an affine relation between the absolute Banzhaf and Rae indices; see Dubey and Shapley (1979). Thus, the Rae index can be given by the following formula:

$$
R_{i}(W)=\frac{1}{2}+\frac{2\left|W_{i}\right|-|W|}{2^{n}}
$$

\subsection{THE KÖNIG AND BRÄUNINGER'S INDEX (OR ZIPKE INDEX)}

Nevison, Zicht, Schoepke in (1978) introduced a power index under the name Zipke index. Then, König and Bräuninger in (1998) reinvented it. In the literature, this index is also called the inclusiveness index, and it can been seen as a measure of success (see, for example, Laruelle, Valenciano, 2011). The König and Bräuninger index, KB, of a simple game $W$ for a player $i$ is defined by:

$$
K B_{i}(W)=\frac{\left|W_{i}\right|}{|W|}
$$

\subsection{THE PUBLIC GOOD INDEX}

The Public Good index (PGI) was defined by Holler in (1982). The PGI considers the coalition value to be a public good. The (relative) PGI of a simple game $W$ for player $i \in N$ is given by:

$$
h_{i}(W)=\frac{\left|W_{i}^{m}\right|}{\sum_{j \in N}\left|W_{j}^{m}\right|}
$$

The PGI index is also called the Holler-Packel index due to the axiomatization of Holler and Packel (1983). Napel in (1999), (2001) showed the independence and non-redundancy of the Holler and Packel axioms.

The absolute Public Good index of simple game $W$ for arbitrary player $i$ is defined as follows:

$$
\bar{h}_{i}(W)=\left|W_{i}^{m}\right|
$$

For the extension of the PGI index to a game value, see Section 5. 


\subsection{THE PUBLIC HELP INDEX $\theta$}

Bertini, Gambarelli and Stach in (2008) introduced the Public Help index (PHI) as a modification of the Public Good index. This index considers that, in assigning a power to a given player $i$, all of the winning coalitions containing player $i$ (unlike the PGI index, which only takes minimal winning coalitions into account). Indeed, sometimes every winning coalition is relevant to the bargaining. The Public Help index, $\theta$ of a non-null simple game $W$ for a player $i \in N$ is given by:

$$
\theta_{i}(W)=\frac{\left|W_{i}\right|}{\sum_{j \in N}\left|W_{j}\right|}
$$

In the case of a null game $W$, this index is $\theta_{i}(W)=0$ for any player $i$. In (Bertini et al., 2008) an axiomatic characterization of the PHI $\theta$ index was provided. For its generalization to a game value, see Section 5 .

The absolute PHI $\theta$ of a simple game $W$ for a player $i \in N$ is the same as the absolute $K B$ index, and is defined for a given simple game $v$ and a player $i \in N$ as the number of all winning coalitions containing player $i$, as follows:

$$
\bar{\theta}_{i}(W)=\overline{K B}_{i}(W)=\left|W_{i}\right|
$$

Note that, after the adequate normalization of the $K B$ index, we obtain the PHI $\theta$ index:

$$
\frac{K B_{i}}{\sum_{j \in N} K B_{j}}=\frac{\left|W_{i}\right|}{\sum_{j \in N}\left|W_{j}\right|}=\theta_{i}(W)
$$

\subsection{THE PUBLIC HELP INDEX $\xi$ (PHI $\xi)$}

In this section, we introduce a new power index PHI $\xi$. The PHI $\xi$ index, like the $K B$ and PHI $\theta$ indices, takes into account all winning coalitions, but it assumes that the probability of forming a winning coalition is inversely proportional to its cardinality and that the players divide the spoils equally in a winning coalition. The Public Help index $\xi$, for a non-null game $W$ and $i \in N$, is defined as follows:

$$
\xi_{i}(W)=\sum_{S \in W_{i}} \frac{1}{|S| \sum_{T \in W} \frac{1}{|T|}} \frac{1}{|S|}=\frac{1}{\sum_{T \in W} \frac{1}{|T|}} \sum_{S \in W_{i}} \frac{1}{|S|^{2}}
$$

In the case of a null game $W$, this index is $\xi_{i}(W)=0$ for any player $i$. Note that each coalition $S$ is formed with probability $\frac{1}{|S| \sum_{T \in W} \frac{1}{|T|}}$, which is inversely proportional to the cardinality of $S$. Therefore, the PHI $\xi$ index can be seen as a hybrid between the PHI index and the Deegan-Packel index.

Justification for introducing the Public Help index $\xi$ is similar to the PHI $\theta$. In assigning the power to players, both indices consider all winning coalitions, not only the minimal wining coalitions as in the PGI. For this reason, $\xi$ and $\theta$ rather describe power 
relations in the consumption of public goods, whereas the PGI analyzes the production of public goods. In production, one has to take care that free-riding is excluded; that is why the PGI considers minimal winning coalitions and in consumption of public goods you cannot avoid free-riding. That is why the Public Help indices give values even to null players. Moreover, $\xi$ (thanks to its formula) gives more power to the winning coalitions with a lower number of members than $\theta$. Thus, the players who contribute to success of less-numerous coalitions obtain more power, and, as a, consequence, null players obtain less power (see Example 4.2).

The absolute PHI $\xi$ of a game $W$ for player $i \in N$, is given by:

$$
\bar{\xi}_{i}(W)=\sum_{S \in W_{i}} \frac{1}{|S|^{2}}
$$

\section{COMPARISON OF POWER INDICES}

In this section, we compare the $K B, \mathrm{PGI}, \mathrm{PHI} \theta, \mathrm{PHI} \xi$, and Rae power indices, taking into account:

- some desirable properties introduced in Section 2,

- the range of power indices, and

- two examples (4.1 and 4.2).

The König and Bräuninger, Rae, and PHI $\theta$ indices are more or less related to the Banzhaf index. The Rae and $K B$ indices satisfy the non-negativity, symmetry, dominance, and bloc postulates but violate the efficiency and null player properties. While the range of values of $R$ and $K B$ indices is the same, and is as follows: $[0.5 ; 1]$. The Public Good index fulfills the efficiency, non-negativity, symmetry, and null postulates but does not satisfy the dominance and bloc properties. All of the above facts written in this paragraph can be found, for example, in (Bertini et al., 2013a).

The index $\theta$ satisfies the efficiency, positivity, and symmetry properties but does not satisfy the null player property. The efficiency and symmetry properties are among the axiomatic characterization of the PHI $\theta$; see (Bertini et al., 2008). Felsenthal and Machover in (1995) demonstrated that, if an index satisfies transfer property, then it also satisfies the dominance postulate. The PHI $\theta$ does not satisfy transfer property (see Bertini et al., 2013a), but it satisfies the dominance property (see Theorem 4.1).

Theorem 4.1. PHI $\theta$ satisfies the dominance property for any weighted game $v \in S_{N}$.

Proof. Consider an arbitrary weighted majority game $v:\left[q ; w_{1}, \ldots, w_{n}\right]$ and two distinct players $i, j \in N$ with weights $w_{i}, w_{j}$ such that $w_{i} \geq w_{j}$. Note that $W_{i}$ (and also $W_{j}$ ) includes a non-empty subset, $W_{i \cup j}$, of all winning coalitions that contain players $i$ and $j$. Namely, $W_{i \cup j}=\{S \in W: i \in S \wedge j \in S\}$ and $W_{i \cup j} \subset W_{i}$ and $W_{i \cup j} \subset W_{j}$. If $w_{i} \geq w_{j}$ then for any non-empty coalition $S \in W_{j} \backslash W_{i \cup j}$ (i.e., $i \notin S$ ), we have $(S \backslash\{j\}) \cup\{i\} \in W_{i}$ : thus, $\left|W_{i}\right| \geq\left|W_{j}\right|$. 
From this, we immediately attain that PHI satisfies the dominance postulate:

$$
\theta_{i}(W)=\frac{\left|W_{i}\right|}{\sum_{k \in N}\left|W_{k}\right|} \geq \frac{\left|W_{j}\right|}{\sum_{k \in N}\left|W_{k}\right|}=\theta_{j}(W)
$$

Kurz in (2014) estimated that the individual power of a player $i \in N$ calculated by $\theta$ is in the following range: $\frac{1}{2 n} \leq \theta_{i}(v) \leq \frac{2}{n}$ for any simple game $v$. In this paper, we show that such an interval is narrower (see Theorem 4.2).

Theorem 4.2. For any simple game $v \in S_{N}$, we have $\frac{1}{2 n-1} \leq \theta_{i}(v) \leq \frac{2}{n+1}$ for any $i \in N$.

Proof. Consider a simple game $v$ with $N$ and an arbitrary player $i \in N$. Let us split the set of all winning coalitions into two distinct sets: $W=W_{i} \cup\left(W \backslash W_{i}\right)$. Thus, $|W|=\left|W_{i}\right|+\left|W \backslash W_{i}\right|, \sum_{j \in N}\left|W_{j}\right|=\sum_{S \in W}|S|=\sum_{S \in W_{i}}|S|+\sum_{S \in\left(W \backslash W_{i}\right)}|S|$, and:

$$
\theta_{i}(W)=\frac{\left|W_{i}\right|}{\sum_{S \in W_{i}}|S|+\sum_{S \in\left(W \backslash W_{i}\right)}|S|}
$$

We remark that, for any simple game, if $S \in\left(W \backslash W_{i}\right)$ then $S \cup\{i\} \in W_{i}$ which implies $\left|W_{i}\right| \geq\left|W \backslash W_{i}\right|$ and, as a consequence, also $\sum_{S \in W_{i}}|S| \geq \sum_{S \in\left(W \backslash W_{i}\right)}|S|$.

Firstly, we demonstrate that the minimal power that an arbitrary player $i$ can obtain in a simple game is equal to $\frac{1}{2 n-1}$. The PHI index $\theta$ for player $i$ has a minimal value if the denominator of (1) attains a maximal value and the numerator of (1) attains a minimal value. The maximal value of denominator (1) is attain for maximal values of both summands $\sum_{S \in W_{i}}|S|$ and $\sum_{S \in\left(W \backslash W_{i}\right)}|S|$. The summand $\sum_{S \in\left(W \backslash W_{i}\right)}|S|$ attains a maximal value when for any $S \in W_{i}$ also $S \backslash\{i\} \in\left(W \backslash W_{i}\right)$.

In this case, we have $\sum_{S \in\left(W \backslash W_{i}\right)}|S|=\sum_{S \in W_{i}}(|S|-1)=\sum_{S \in W_{i}}|S|-\left|W_{i}\right|$, and we can rewrite (1) as follows:

$$
\theta_{i}(W)=\frac{\left|W_{i}\right|}{2 \sum_{S \in W_{i}}|S|-\left|W_{i}\right|}
$$

Since $v(N)=1$ for any non-null game $v \in S_{N}$, we see that the minimal value of $\left|W_{i}\right|$ is equal to 1 for any $i \in N$. Suppose that $\left|W_{i}\right|=1$. Thus, we have that $\sum_{S \in W_{i}}|S|=|N|=n$. Now, in (2), substituting 1 for $\left|W_{i}\right|$ and $n$ for $\sum_{S \in W_{i}}|S|$, we conclude:

$$
\frac{1}{2 n-1} \leq \theta_{i}(W) \text { for any } i \in N
$$

Now, let us demonstrate that the maximal power that $\theta$ can assign to a player $i \in N$ is equal to $\frac{2}{n+1}$. The PHI index $\theta$ for player $i$ has a maximal value if the denominator of (1) attains the minimal value and the numerator of (1) attains a maximal value. 
The denominator of (1) attains its minimum value if $\sum_{S \in\left(W \backslash W_{i}\right)}|S|=0$. Whereas, $\left|W_{i}\right|$ (i.e., numerator of (1)) attains its maximum value when all coalitions with player $i$ are winning (it also means that player $i$ is a dictator). Since there are $2^{n-1}$ coalitions that contain player $i$, we see that the maximum value of numerator (1) is equal to $\left|W_{i}\right|=2^{n-1}$ and $\sum_{S \in W_{i}}|S|=\sum_{k=1}^{n} k\left(\begin{array}{c}n-1 \\ k-1\end{array}\right)$. Applying, for example, the binomial identity $(1+x)^{n}=\sum_{k=0}^{n}\left(\begin{array}{l}n \\ k\end{array}\right) x^{k} \forall x \in R$, it could be proven that $\sum_{k=1}^{n} k\left(\begin{array}{l}n-1 \\ k-1\end{array}\right)=(n+1) 2^{n-2}$ (for a full demonstration, see the Appendix). Now replacing in (1) $\left|W_{i}\right|$ with $2^{n-1}, \sum_{S \in W_{i}}|S|$ with $(n+1) 2^{n-2}$ and $\sum_{S \in W \backslash W_{i}}|S|$ with 0 , we immediately attain $\theta_{i}(W) \leq \frac{\left|W_{i}\right|}{\sum_{S \in W_{i}}|S|}=\frac{2^{n-1}}{(n+1) 2^{n-2}}=\frac{2}{(n+1)}$.

Let us consider the new index $\xi$ introduced in Section 3.5. We will prove that the newly proposed index PHI $\xi$ satisfies the following properties: efficiency, non-negativity, symmetry, and dominance (see Theorems 4.3-4.6).

Theorem 4.3. PHI $\xi$ satisfies the efficiency postulate:

$$
\sum_{i \in N} \xi_{i}(W)= \begin{cases}1 & \text { if } W \text { is not the null game } \\ 0 & \text { otherwise. }\end{cases}
$$

Proof. Let $W$ be a game with a set of players $N$. If $W$ is a null game, each player is a zero player, so $\sum_{i \in N} \xi_{i}(W)=0$. While for a non-null game $W$, we attain:

$$
\begin{aligned}
& \sum_{i \in N} \xi_{i}(W)=\sum_{i \in N}\left(\frac{1}{\sum_{T \in W} \frac{1}{|T|}} \sum_{S \in W_{i}} \frac{1}{|S|^{2}}\right)=\frac{1}{\sum_{T \in W} \frac{1}{|T|}} \sum_{i \in N}\left(\sum_{S \in W_{i}} \frac{1}{|S|^{2}}\right) \\
&=\frac{1}{\sum_{T \in W} \frac{1}{|T|}}\left(\sum_{S \in W_{1}} \frac{1}{|S|^{2}}+\sum_{S \in W_{2}} \frac{1}{|S|^{2}}+\ldots+\sum_{S \in W_{n}} \frac{1}{|S|^{2}}\right) \\
&= \frac{1}{\sum_{T \in W} \frac{1}{|T|}} \sum_{S \in W}|S| \frac{1}{|S|^{2}}=\frac{1}{\sum_{T \in W} \frac{1}{|T|}} \sum_{S \in W} \frac{1}{|S|}=1 \\
& F(x)=\frac{\sqrt{x-1}^{2}}{x^{4}}
\end{aligned}
$$

Theorem 4.4. For any simple game $W$ and for any $i \in N$, we have $\xi_{i}(W) \geq 0$.

Proof. The PHI $\xi$ of a non-null simple game $W$ and a player $i \in N$ is always greater than zero. It is consequential that, in any non-null game, at least one winning coalition exists (i.e., grand coalition $N$ ). Thus, $N \in W \neq \emptyset$ and $N \in W_{i} \neq \emptyset$, and, as a consequence, we have $\xi_{i}(W)=\frac{1}{\sum_{T \in W} \frac{1}{|T|}} \sum_{S \in W_{i}} \frac{1}{|S|^{2}}>0$. In the case of a null game $\xi_{i}(W)=0$ $\forall i \in N$ since, in a null game, all players are zero players. 
Theorem 4.5. For any simple game W, PHI $\xi$ satisfies the symmetry postulate.

Proof. Let us fix a simple game $W$. It is sufficient to prove that $\xi_{i}(W)=\xi_{\pi(i)}(\pi(W))$ for each $i \in N$ and all permutations $\pi: N \rightarrow N$. In case of a null game, it is straightforward to prove that symmetry holds since, in a null game, each player is zero player. In the case of a non-null game $W$, we have:

$$
\xi_{i}(W)=\frac{1}{\sum_{T \in W} \frac{1}{|T|}} \sum_{S \in W_{i}} \frac{1}{|S|^{2}}=\frac{1}{\sum_{T \in W} \frac{1}{|T|}} \sum_{S \in W_{\pi(i)}} \frac{1}{|S|^{2}}=\xi_{\pi(i)}(W)
$$

Theorem 4.6. For any simple game $W$, PHI $\xi$ satisfies the dominance postulate.

Proof. Consider an arbitrary weighted majority game $\left[q ; w_{1}, \ldots, w_{n}\right]$ and two distinct players $i, j \in N$ with weights $w_{i}, w_{j}$ respectively such that $w_{i} \geq w_{j}$. As in the proof of Theorem 4.1, we can show that, if $w_{i} \geq w_{j}$, then $\left|W_{i}\right| \geq\left|W_{j}\right|$, and if $W_{j} \backslash W_{i} \neq \emptyset$, then for any winning coalition $S \in\left(W_{j} \backslash W_{i}\right)$, the coalition $(S \backslash\{j\}) \cup\{i\} \in\left(W_{i} \backslash W_{j}\right)$, and $|S|=|(S \backslash\{j\}) \cup\{i\}|$. Hence, we have not only that $\left|W_{i} \backslash W_{j}\right| \geq\left|W_{j} \backslash W_{i}\right|$, but also $\sum_{S \in W_{i} \backslash W_{j}} \frac{1}{|S|^{2}} \geq \sum_{S \in W_{j} \backslash W_{i}} \frac{1}{|S|^{2}}$; as a consequence, we immediately attain that $\xi$ satisfies the dominance postulate:

$$
\begin{aligned}
\xi_{i}(W)=\frac{\sum_{S \in W_{i}} \frac{1}{|S|^{2}}}{\sum_{T \in W} \frac{1}{|T|}} & =\frac{\sum_{S \in W_{i} \backslash W_{j}} \frac{1}{|S|^{2}}+\sum_{S \in W_{i}} \cap W_{j} \frac{1}{|S|^{2}}}{\sum_{T \in W} \frac{1}{|T|}} \\
& \geq \frac{\sum_{S \in W_{j} \backslash W_{i}} \frac{1}{|S|^{2}}+\sum_{S \in W_{i}} \cap W_{j} \frac{1}{|S|^{2}}}{\sum_{T \in W} \frac{1}{|T|}}=\frac{\sum_{S \in W_{j}} \frac{1}{|S|^{2}}}{\sum_{T \in W} \frac{1}{|T|}}=\xi_{j}(W)
\end{aligned}
$$

Example 4.1. Let us consider a game given by the following characteristic function: $v(\{1\})=0, v(\{2\})=0, v(\{3\})=0, v(\{2,3\})=0, v(\{1,2\})=1, v(\{1,3\})=1$, $v(\{1,2,3\})=1$. In Table 1 , we present the payoffs assigned by the Rae, König and Bräuninger, PGI, PHI $\theta$ and $\xi$ indices to players in the considered game.

Table 1. Distribution of power in Example 4.1

\begin{tabular}{|c|c|c|c|}
\hline Power index & Player 1 & Player 2 & Player 3 \\
\hline$R$ & $7 / 8$ & $5 / 8$ & $5 / 8$ \\
\hline$K B$ & 1 & $2 / 3$ & $2 / 3$ \\
\hline$h$ & $1 / 2$ & $1 / 4$ & $1 / 4$ \\
\hline$\theta$ & $3 / 7$ & $2 / 7$ & $2 / 7$ \\
\hline$\xi$ & $22 / 48$ & $13 / 48$ & $13 / 48$ \\
\hline
\end{tabular}

Example 4.2. Let us consider a game $W=\{\{1,2\},\{1,3\},\{1,2,3\},\{1,2,4\}$, $\{1,3,4\},\{1,2,3,4\}\}$. In this game, there are only two minimal winning coalitions: $\{1,2\},\{1,3\}$. In Table 2 , we present the payments assigned by the considered five power indices to the players. 
Table 2. Distribution of power in Example 4.2

\begin{tabular}{|c|c|c|c|c|}
\hline Power index & Player 1 & Player 2 & Player 3 & Player 4 \\
\hline$R$ & $7 / 8$ & $5 / 8$ & $5 / 8$ & $1 / 2$ \\
\hline$K B$ & 1 & $2 / 3$ & $2 / 3$ & $1 / 2$ \\
\hline$h$ & $1 / 2$ & $1 / 4$ & $1 / 4$ & 0 \\
\hline$\theta$ & $6 / 17$ & $4 / 17$ & $4 / 17$ & $3 / 17$ \\
\hline$\xi$ & $129 / 324$ & $77 / 324$ & $77 / 324$ & $41 / 324$ \\
\hline
\end{tabular}

In Example 4.2 (Table 2), we can observe that PHI $\xi$ violates the null property.

As we can observe in Examples 4.1 and 4.2, the power indices taken into account in this paper split the total wins in different ways and assign different power to the players, but give the same rankings to the players. An interesting fact is that some of these power indices induce the same rankings of players, not only in the considered examples, but also in any simple game. Namely, the König and Bräuninger, PHI $\theta$, and Rae indices rank players in the same way. Moreover, they give the same rankings as the Banzhaf power index, since, for a given game $W$ and a player $i$, all of these indices $\left(K B, \theta, R\right.$, and Banzhaf indices) are positive affine transformations of $\left|W_{i}\right|$ (see Section 3 and (Bertini et al., 2013a)).

The bloc property is one of the most important properties necessary for power indices to be useful for analysis of block-expansion mechanisms in the decision-making bodies (see, for example, (Felsenthal and Machover, 1995; Jasiński, 2013)). The KB and Rae indices satisfy bloc property, whereas the PGI index does not fulfill this property (see (Bertini et al., 2013a)).

In Table 3, we summarize all results discussed in this section.

Table 3. Power indices $R, K B, h, \theta$, $\xi$ in comparison

\begin{tabular}{|l|c|c|c|c|c|}
\hline \multirow{2}{*}{\multicolumn{1}{|c|}{ Property }} & \multicolumn{5}{c|}{ Power index } \\
\cline { 2 - 6 } & $R$ & $K B$ & $h$ & $\theta$ & $\xi$ \\
\hline Bloc & yes & yes & no & $?$ & $?$ \\
\hline Dominance & yes & yes & no & yes & yes \\
\hline Efficiency & $n o$ & $n o$ & yes & yes & yes \\
\hline Non-negativity & yes & yes & yes & yes & yes \\
\hline Null player & $n o$ & $n o$ & yes & no & no \\
\hline Symmetry & yes & yes & yes & yes & yes \\
\hline $\begin{array}{l}\text { Range of power index in } \\
\text { a non-null game } v, n \geq 2\end{array}$ & {$[0.5 ; 1]$} & {$[0.5 ; 1]$} & {$[0 ; 1]$} & {$\left[\frac{1}{2 n-1} ; \frac{2}{n+1}\right]$} & $?$ \\
\hline
\end{tabular}

By "?", we denote that it is still an open problem. 


\section{EXTENSIONS OF THE PHI INDICES TO THE GAME VALUES}

In this section, we propose the extensions of the PHI indices ( $\theta$ and $\xi$ ) to general cooperative games. But we start with introducing the Public Good value.

Holler and $\mathrm{Li}$ in (1995) extended the PGI index to the Public Good value introducing the concept of a real gaining coalition (RGC).

Definition 5.1. For a cooperative game $(N, v)$, a subset $S \subseteq N$ is called a real gaining coalition (RGC) if, for any $T \subset S$, we have $v(S)-v(T)>0$.

Let $R G C(v)$ denote a set of all real gaining coalitions in the game $v$, and by $R G C_{i}(v)$ (or $R G C_{i}$ ), we denote a set of all real gaining coalitions containing player $i$. In a cooperative game, the concept of the real gaining coalition corresponds with the concept of the minimal winning coalition in a simple game. Moreover, for any simple game $v$, we have $W^{m}(v)=R G C(v)$.

Definition 5.2. A player $i \in N$ is a dummy player if he does not belong to any $S \in R G C(v)$.

In a general cooperative game, the concept of a dummy player corresponds with the concept of a null player in a simple game. Therefore, similarly like in a simple game, in any minimal winning coalition, all players contribute to its win, in general cooperative game we have that if and only if all players contribute to the worth of a coalition, the coalition is a RGC.

Holler and Li (1995) defined the Public Good value and its normalized version, only taking into account the payoffs from real gaining coalitions (see Definition 5.3 and 5.4).

Definition 5.3. The Public Good value (or the Holler value) of cooperative game $v$ for a player $i \in N$ is given by: $\overline{H V}_{i}(v)=\bar{h}_{i}(v)=\sum_{S \in R G C_{i}} v(S)$.

Normalizing the PGV value to the coalition payoff of the grand coalition $N$, we obtain a normalized version of PGV.

Definition 5.4. The normalized Public Good value (or the normalized Holler value) of cooperative game $v$ for a player $i \in N$ is given by: $H V_{i}(v)=h_{i}(v)=\frac{\bar{h}_{i}(v)}{\sum_{j \in N} \bar{h}_{j}(v)} v(N)$.

We remark that Holler and $\mathrm{Li}$ in (1995) axiomatized the Holler value with four axioms: L1 axiom (efficiency), L2 axiom (mergeability), symmetry, and dummy player.

Following the idea of Holler and Li (1995), we propose the extensions of the PHI indices $\theta$ and $\xi$ from simple games to general ones in Sections 5.1 and 5.2. In order to state the definitions of the announced values, we need to first introduce the concept of a gaining coalition (GC).

Definition 5.5. For a cooperative game $(N, v)$, a subset $S \subseteq N$ is called a gaining coalition $(\mathrm{GC})$ if $v(S)>0$. 
Let $G C(v)$ denote a set of all gaining coalitions in the game $v$, and by $G C_{i}(v)$ (or $G C_{i}$ ), we denote a set of all gaining coalitions containing player $i$. We remark that the concept of the gaining coalition in a general cooperative game is equivalent to the concept of the winning coalition in a simple game. Furthermore, for any simple game $v$, we have $W(v)=G C(v)$.

\subsection{PUBLIC HELP VALUE $\boldsymbol{\theta}$}

Let us introduce an extension of the PHI $\theta$ into a general game, considering only the payoffs from GCs.

Definition 5.6. The absolute Public Help value $\bar{\theta}$ (or the $\overline{K B}$ value) of cooperative game $v$ for a player $i \in N$ is given by $\bar{\theta}_{i}(v)=\sum_{S \in G C_{i}} v(S)$ if a coalition $S \subseteq N$ exists such that $v(S)>0$, otherwise $\bar{\theta}_{i}(v)=0$.

We also propose the normalized (to the coalition payoff of grand coalition $N$ ) version of the PHV value.

Definition 5.7. The normalized Public Help value $\theta$ (or the $K B$ value) of cooperative game $v$ for a player $i \in N$ is given by $\theta_{i}(v)=\frac{\bar{\theta}_{i}(v)}{\sum_{j \in N} \bar{\theta}_{j}(v)} v(N)$ if a coalition $S \subseteq N$ exists such that $v(S)>0$, otherwise $\theta_{i}(v)=0$.

Now, we prove that absolute and normalized Public Help values $\bar{\theta}$ and $\theta$ satisfy several properties. Namely, in Theorem 5.1, we prove that these values assign nonnegative payments to players. In Theorem 5.2, we show that the total gain $v(N)$ is distributed by the normalized PHV $\theta$. And finally, Theorem 5.3 states that $\bar{\theta}$ and $\theta$ are symmetric, which means that "symmetric" players received the same payment.

Theorem 5.1. For any cooperative game $(v, N)$ and for any $i \in N$, we have $\bar{\theta}_{i}(v) \geq 0$ and $\theta_{i}(v) \geq 0$.

Proof. Let $(v, N)$ be a cooperative game. If a coalition $S \subseteq N: v(S)>0$ does not exist, then directly from Definitions 5.6 and 5.7, we have $\bar{\theta}_{i}(v)=\theta(v)=0$. Otherwise, if a coalition $S \subseteq N$ exists such that $v(S)>0$, then $v(N)>0$, since $v$ is a monotonic game and either $|G C|>0$ and $\left|G C_{i}\right|>0$ for any $i \in N$. Thus, for any $i \in N$, we have $\bar{\theta}_{i}(v)=\sum_{S \in G C_{i}} v(S)>0$ and $\theta_{i}(v)=\frac{\bar{\theta}_{i}(v)}{\sum_{j \in N} \bar{\theta}_{j}(v)} v(N)>0$, which completes the proof.

Theorem 5.2. For any cooperative game $(v, N)$, we have $\sum_{i \in N} \theta_{i}(v)=v(N)$.

Proof. Let $(v, N)$ be a cooperative game. If a coalition $S \subseteq N$ exists such that $v(S)>0$, then $v(N)>0$, since $v$ is a monotonic game and either $|G C|>0$ and $\left|G C_{i}\right|>0$ for any $i \in N$. Thus, for any $i \in N$, we have $\sum_{i \in N} \theta_{i}(v)=\sum_{i \in N} \frac{\sum_{S \in G C_{i}} v(S)}{\sum_{j \in N} \sum_{S \in G C_{j}} v(S)} v(N)=$ $v(N) \frac{\sum_{i \in N} \sum_{S \in G C_{i}} v(S)}{\sum_{j \in N} \sum_{S \in G C_{j}} v(S)}=v(N)$. Otherwise, if a coalition $S \subseteq N: v(S)>0$ does not exist, then $v(N)=0$, and from Definition 5.7, we see that $\theta_{i}(v)=0$ which completes the proof. 
Theorem 5.3. For any cooperative game $v, \bar{\theta}_{i}(v)=\bar{\theta}_{\pi(i)}(\pi(v))$ and $\theta_{i}(v)=\theta_{\pi(i)}(\pi(v))$ for each $i \in N$ and all permutations $\pi: N \rightarrow N$.

Proof. Let $(v, N)$ be a cooperative game, $i$ be an arbitrary player, and $\pi$ a permutation on $N$. In case of a null game, we have that all players received zero (directly from Definitions 5.6 and 5.7). Thus, the theorem holds. In case of a non-null game, we have: $\bar{\theta}_{i}(v)=\sum_{S \in G C_{i}} v(S)=\sum_{S \in G C_{\pi(i)}} v(S)=\bar{\theta}_{\pi(i)}(v)$ and $\theta_{i}(v)=\frac{\bar{\theta}_{i}(v)}{\sum_{j \in N} \bar{\theta}_{j}(v)} v(N)=$ $\frac{\bar{\theta}_{\pi(i)}(v)}{\sum_{\pi(j) \in N} \bar{\theta}_{\pi(j)}(v)} v(N)=\theta_{\pi(i)}(v)$.

\subsection{PUBLIC HELP VALUE $\xi$}

Let us introduce an extension of the PHI $\xi$ into the general game. The Public Help value $\xi(\mathrm{PHV} \xi)$, like $\mathrm{PHV} \theta$, only regards payoffs from GCs.

Definition 5.8. The absolute Public Help value $\bar{\xi}$ of cooperative game $v$ for a player $i \in N$ is given by $\bar{\xi}_{i}(v)=\sum_{S \in G C_{i}} \frac{v(S)}{|S|^{2}}$ if a coalition $S \subseteq N$ exists such that $v(S)>0$, otherwise $\bar{\xi}_{i}(v)=0$.

We also introduce the normalized (to the coalition payoff of grand coalition $N$ ) version of the $\mathrm{PHV} \xi$ value:

Definition 5.9. The normalized Public Help value $\xi$ of cooperative game $v$ for a player $i \in N$ is given by $\xi_{i}(v)=\frac{v(N)}{\sum_{S \in G C} \frac{v(S)}{|S|}} \bar{\xi}_{i}(v)$ if a coalition $S \subseteq N$ exists such that $v(S)>0$, otherwise $\xi_{i}(v)=0$.

We state and prove that the absolute and normalized PHVs $\bar{\xi}$ and $\xi$ satisfy the properties considered for $\bar{\theta}$ and $\theta$ in Section 5.1. This means the extended values $\bar{\xi}$ and $\xi$ preserve not only non-negativity (Theorem 5.4), but also the symmetry (Theorem 5.6).

Theorem 5.4. For any cooperative game $v$ and for any $i \in N$, we have $\bar{\xi}_{i}(v) \geq 0$ and $\xi_{i}(v) \geq 0$.

Proof. Let $(v, N)$ be a cooperative game. If a coalition $S \subseteq N: v(S)>0$ does not exist, then we have $\bar{\xi}_{i}(v)=\xi(v)=0$ (directly from Definitions 5.8 and 5.9). Otherwise, if a coalition $S \subseteq N$ exists such that $v(S)>0$, then $v(N)>0$, since $v$ is a monotonic game and either $|G C|>0$ and $\left|G C_{i}\right|>0$ for any $i \in N$. Thus, for any $i \in N$, we have $\bar{\xi}_{i}(v)=\sum_{S \in G C_{i}} \frac{v(S)}{|S|^{2}}>0$ and $\xi_{i}(v)=\frac{v(N)}{\sum_{S \in G C} \frac{v(S)}{|S|}} \sum_{S \in G C} \frac{v(S)}{|S|}>0$, which completes the proof.

Theorem 5.5. For any cooperative game $(v, N)$, we have $\sum_{i \in N} \xi_{i}(v)=v(N)$. 
Proof. Let $(v, N)$ be a cooperative game. If a coalition $S \subseteq N$ exists such that $v(S)>0$, then $v(N)>0$, since $v$ is a monotonic game and either $|G C|>0$ and $\left|G C_{i}\right|>0$ for any $i \in N$.

Thus, for any $i \in N$, we have:

$$
\begin{aligned}
\sum_{i \in N} \xi_{i}(v) & =\sum_{i \in N} \frac{v(N) \sum_{S \in G C_{i}} \frac{v(S)}{|S|^{2}}}{\sum_{S \in G C} \frac{v(S)}{|S|}}=v(N) \frac{\sum_{i \in N} \sum_{S \in G C_{i}} \frac{v(S)}{|S|^{2}}}{\sum_{S \in G C} \frac{v(S)}{|S|}} \\
& =v(N) \frac{\sum_{S \in G C}|S| \frac{v(S)}{|S|^{2}}}{\sum_{S \in G C} \frac{v(S)}{|S|}}=v(N) \frac{\sum_{S \in G C} \frac{v(S)}{|S|}}{\sum_{S \in G C} \frac{v(S)}{|S|}}=v(N)
\end{aligned}
$$

Otherwise, if a coalition $S \subseteq N: v(S)>0$ does not exist, then $v(N)=0$; and from Definition 5.9, we have that $\xi_{i}(v)=0$, which completes the proof.

It is not difficult to prove that both values $\bar{\xi}$ and $\xi$ allocate equal payments to symmetric players (see Theorem 5.6).

Theorem 5.6. For any cooperative game $v, \bar{\xi}_{i}(v)=\bar{\xi}_{\pi(i)}(\pi(v))$ and $\xi_{i}(v)=\xi_{\pi(i)}(\pi(v))$ where $(\pi(v))(S)=v\left(\pi^{-1}(S)\right)$ for every $i \in N$ and all permutations $\pi: N \rightarrow N$.

Proof. Let $(v, N)$ be a cooperative game, $i$ be an arbitrary player, and $\pi$ a permutation on $N$. In case of a null game, we see that all players received zero (directly from definitions 5.8 and 5.9). Thus, the theorem holds. In case of a non-null game, we have:

$$
\bar{\xi}_{i}(v)=\sum_{S \in G C_{i}} \frac{v(S)}{|S|^{2}}=\sum_{S \in G C_{\pi(i)}} \frac{v(S)}{|S|^{2}}=\bar{\xi}_{\pi(i)}(v)
$$

and

$$
\xi_{i}(W)=\frac{v(N)}{\sum_{S \in G C} \frac{1}{|S|}} \bar{\xi}_{i}(v)=\frac{v(N)}{\sum_{S \in G C} \frac{1}{|S|}} \bar{\xi}_{\pi(i)}(v)=\xi_{\pi(i)}(v)
$$

\section{CONCLUSION AND FURTHER DEVELOPMENTS}

In this paper, we analyzed from a different point of view some values and power indices well-defined in the social context where the goods are public. We consider the Public Good index (Holler, 1982), the Public Good value (Holler and Li, 1995), the Public Help index (Bertini et al., 2008), the König and Bräuninger index (1998) (see also (Nevison et al. 1978; Nevison, 1979)), and the Rae index (1969). The aims of this paper were as follows: to propose an extension of the Public Help index to cooperative games; to introduce a new power index with its extension to a game value; and to provide some characterizations of the new index and values.

It is easy to see that the results shown in this paper are not exhaustive. As developments can be many, we simply indicate that those may be of some interest (in our humble opinion). Namely, the new index (PHI $\xi$ ) and two new values (PHV $\theta$ and $\mathrm{PHV} \xi$ ) introduced needed axiomatic derivations. Then, the algorithms for automatic computation of new index and new values could be supplied. We suspect that Public 
Help indices $\theta$ and $\xi$ satisfy the bloc property, but it is still an open problem. The new power index could be compared to all of the other indices, taking into account other properties; for example, those analyzed in (Felsenthal and Machover, 1998; Bertini et al., 2013a, 2013b). Still, regarding $\xi$, it might be of some interest to analyze its rankings and compare them with the rankings of other indices.

Last but not least, the new values and PHI indices could be extended to games with a priori unions, with incompatibilities, with affinities, or with various probabilities of coalition formation (see e.g., Fragnelli et al., 2009).

\section{ACKNOWLEDGMENTS}

The authors would like to thank the anonymous reviewers for their valuable comments and suggestions to improve the quality of this paper.

\section{APPENDIX}

Here we present proof of the following identity:

$$
\sum_{k=1}^{n} k\left(\begin{array}{l}
n-1 \\
k-1
\end{array}\right)=(n+1) 2^{n-2} \quad \forall n \geq 2
$$

The identity (3) can be proven starting with the binomial identity:

$$
(1+x)^{n}=\sum_{k=0}^{n}\left(\begin{array}{l}
n \\
k
\end{array}\right) x^{k} \text { for any real number } x \text { and } n \geq 1
$$

Since $\left(\begin{array}{l}n \\ k\end{array}\right)=\frac{n}{k}\left(\begin{array}{l}n-1 \\ k-1\end{array}\right)$ for $n \geq k>0$, the above binomial identity can be rewritten as:

$$
(1+x)^{n}=1+n \sum_{k=1}^{n} \frac{1}{k}\left(\begin{array}{l}
n-1 \\
k-1
\end{array}\right) x^{k} \text { for any real number } x
$$

Taking the derivative of the both parts of (4) with respect to $x$, we attain:

$$
n(1+x)^{n-1}=n \sum_{k=1}^{n}\left(\begin{array}{l}
n-1 \\
k-1
\end{array}\right) x^{k-1}
$$

Substituting 1 for $x$, we obtain the following identity:

$$
\sum_{k=1}^{n}\left(\begin{array}{l}
n-1 \\
k-1
\end{array}\right)=2^{n-1} \quad \forall n \geq 2
$$


Now, taking the derivative of the both parts of (5) with respect to $x$, we attain $(n-1)(1+x)^{n-2}=\sum_{k=1}^{n}(k-1)\left(\begin{array}{l}n-1 \\ k-1\end{array}\right) x^{k-2}$. Substituting 1 for $x$, we have:

$$
(n-1) 2^{n-2}=\sum_{k=1}^{n}(k-1)\left(\begin{array}{l}
n-1 \\
k-1
\end{array}\right)
$$

From (7), we can calculate $\sum_{k=1}^{n} k\left(\begin{array}{l}n-1 \\ k-1\end{array}\right)$; and using identity (6), we conclude:

$$
\sum_{k=1}^{n} k\left(\begin{array}{l}
n-1 \\
k-1
\end{array}\right)=(n-1) 2^{n-2}+\sum_{k=1}^{n}\left(\begin{array}{l}
n-1 \\
k-1
\end{array}\right)=(n-1) 2^{n-2}+2^{n-1}=(n+1) 2^{n-2}
$$

\section{REFERENCES}

Banzhaf, J.F., 1965. Weighted voting doesn't work: A mathematical analysis. Rutgers Law Review, 19, pp. 317-343.

Bertini, C., Freixas, J., Gambarelli, G., Stach, I., 2013a. Comparing power indices. In: V. Fragnelli, G. Gambarelli (eds), Open Problems in the Theory of Cooperative Games A Special Issue of Int. Game Theory Review, 15(2), pp. 1340004-1-1340004-19.

Bertini, C., Freixas, J., Gambarelli, G., Stach, I., 2013b. Some open problems in simple games. In: V. Fragnelli, G. Gambarelli (eds), Open Problems in the Theory of Cooperative Games - A Special Issue of Int. Game Theory Review, 15(2), pp. 1340005-1-1340005-18.

Bertini, C., Gambarelli, G., Stach, I., 2008. A public help index. In: M. Braham, F. Steffen (eds), Power, Freedom, and Voting, pp. 83-98, Springer Verlag.

Brams, S. and Lake, M., 1978. Power and satisfaction in a representative democracy. In: P.C. Ordenshook (ed.), Game Theory and Political Science, pp. 529-562. New York University Press.

Coleman, J.S., 1971. Control of collectivities and the power of collectivity to act. In: B. Lieberman (ed.), Social Choice, pp. 269-300. Gordon and Breach, New York.

Deegan, J., Packel, E.W., 1978. A new index of power for simple n-person games, International Journal of Game Theory, 7, pp. 113-123.

Dubey, P., Shapley, L., 1979. Mathematical properties of the Banzhaf power index, Mathematics of Operations Research, 4(2), pp. 99-131.

Felsenthal, D., Machover, M., 1995. Postulates and paradoxes of relative voting power A critical reappraisal, Theory and Decision, 38, pp. 195-229.

Felsenthal, D., Machover, M., 1998. The measurement of voting power. Theory and practice, problems and paradoxes. Edward Elgar Publishing, Cheltenham.

Fragnelli, V., Ottone, S., Sattanino, R., 2009. A New Family of Power Indices for Voting Games, Homo Oeconomicus, 26, pp. 381-392.

Holler, M.J., 1982. Forming coalitions and measuring voting power, Political Studies, 30, pp. 262-271.

Holler, M.J., Li, X., 1995. From public good index to public value. An axiomatic approach and generalization. Control and Cybernetics, 24(3), pp. 257-270.

Holler, M.J., Packel, E.W., 1983. Power, luck and the right index, Zeitschrift für Nationalökonomie, Journal of Economics, 43, pp. 21-29. 
Jasiński, M., 2013. The terms of cooperation's stability. What is the reason of flow between coalitions? Roczniki Kolegium Analiz Ekonomicznych [Annals of the Collegium of Economic Analysis], 32, pp. 55-76.

Johnston, R., 1978. On the measurement of power: Some reactions to Laver. Environment and Planning A, 10(8), pp. 907-914.

König, T., Bräuninger, T., 1998. The inclusiveness of European decision rules. Journal of Theoretical Politics, 10, pp. 125-142.

Kurz, S., 2014. The inverse problem for power distributions in committees. arXiv.org, arXiv:1402.0988. Retrieved from http://arxiv.org/abs/1402.0988 February 2015.

Laruelle, A., Valenciano, F., 2011. Voting and Collective Decision-Making. Cambridge Books. Cambridge University Press.

Mercik, J.W., 1997. Power and expectations. Control and Cybernetics, 26(4), pp. 617-621.

Napel, S., 1999. The Holler-Packel axiomatization of the public good index completed. Homo Oeconomicus, 15, pp. 513-520.

Napel, S., 2001. A Note on the Holler-Packel Axiomatization of the Public Good Index (PGI). In: M.J. Holler, G. Owen (eds), Power Indices and Coalition Formation, Kluwer, pp. $143-151$.

Nevison, Chr.H., Zicht, B., Schoepke, S., 1978. A naive approach to the Banzhaf index of power. Behavioral Science, 23(2), pp. 130-131.

Nevison Chr.H., 1979. Structural power and satisfaction in simple games. Applied Game Theory, Physica-Verlag, Wuerzburg, pp. 39-57.

Rae, D., 1969. Decision rules and individual values in constitutional choice. American Political Science Review, 63, pp. 40-56.

Shapley, L.S., 1953. A value for n-person games. In: A.W. Tucker, H.W. Kuhn (eds), Contributions to the Theory of Games II, pp. 307-317. Princeton University Press, Princeton.

Shapley, L.S., Shubik, M., 1954. A method for evaluating the distribution of power in a committee system. American Political Science Review, 48, pp. 787-792. 